\title{
Advanced oxidation protein products and serum total oxidant/antioxidant status levels in rosacea
}

\author{
Hilal Kaya Erdogan¹, Isil Bulur², Evin Kocaturk³, Zeynep Nurhan Saracoglu¹, Ozkan Alatas ${ }^{4}$ Muzaffer Bilgin ${ }^{5}$
}

${ }^{1}$ Department of Dermatology, Faculty of Medicine, Eskisehir Osmangazi University, Eskisehir, Turkey

${ }^{2}$ Department of Dermatology, Memorial Sisli Hospital, Istanbul, Turkey

${ }^{3}$ Department of Biochemistry, Eskisehir Yunus Emre State Hospital, Eskisehir, Turkey

${ }^{4}$ Department of Biochemistry, Faculty of Medicine, Eskişehir Osmangazi University, Eskisehir, Turkey

${ }^{5}$ Department of Biostatistics, Faculty of Medicine, Eskişehir Osmangazi University, Eskisehir, Turkey

Adv Dermatol Allergol 2018; XXXV (3): 304-308

DOI: https://doi.org/10.5114/ada.2018.76228

\begin{abstract}
Introduction: Rosacea is a chronic, inflammatory dermatosis which develops due to the effect of genetic and environmental factors.

Aim: To evaluate the oxidative stress in rosacea patients by measuring serum total antioxidant status (TAS), total oxidant status (TOS), oxidative stress index (OSI) and advanced oxidation protein products (AOPP) levels in our study. Material and methods: Our study included rosacea patients and healthy volunteers aged between 18 and 65 years. Total antioxidant status, TOS and AOPP levels were measured and OSI was calculated.

Results: The study included 70 rosacea patients and 30 healthy volunteers as a control group. When TAS, TOS, OSI and AOPP levels were compared between rosacea and control groups, there was no difference for OSI levels; while TAS, TOS and AOPP levels were significantly higher in the rosacea group $(p=0.151, p=0.013, p=0.034, p=0.017$, respectively). In the rosacea group, there was no correlation between TAS, TOS, OSI and AOPP levels and disease duration. Besides there was no difference between family history, rosacea type, symptom frequency and ocular involvement and TAS, TOS, OSI and AOPP levels in the rosacea group.

Conclusions: We observed that serum TAS, TOS and AOPP levels were significantly higher in rosacea patients, but there was no significant difference among the disease activity parameters. These results can support the role of oxidative stress in the pathogenesis of rosacea.
\end{abstract}

Key words: rosacea, oxidative stress, advanced oxidation protein products.

\section{Introduction}

Rosacea is a common, chronic, inflammatory dermatosis which develops by the effect of genetic and environmental factors. It is located on the central face and characterized by erythema, telangiectasia, papules and pustules. Four clinical subtypes were identified by the National Rosacea Society as: erythematotelangiectatic, papulopustular, phymatous, and ocular rosacea [1, 2].

The etiopathogenesis of rosacea is not known exactly; however genetic predisposition, ultraviolet radiation (UV), Demodex colonization, microbial stimuli, abnormalities in the immune system, neurovascular dysregulation and oxidative stress are suggested [1, 2].

In the literature, there are studies investigating the oxidative stress in rosacea patients [3-9]. In order to evalu- ate the oxidative stress status, reactive oxygen species, non-enzymatic molecules and antioxidant enzymes can be measured. Nevertheless they do not reflect global assessment of oxidative stress. Oxidative stress status can be evaluated globally by measuring total antioxidant status (TAS) and total oxidant status (TOS) via a newly developed method [10, 11]. Oxidative modification products of proteins have some advantages such as early formation, longer lifespan, greater stability and reliability in comparison with other oxidative markers. Witko-Sarsat et al. defined a novel oxidative stress marker of protein, referred to as advanced oxidation protein products (AOPP). Advanced oxidation protein products can be used in evaluating the oxidant-mediated protein damage, which occurs only through myeloperoxidase activity of neutrophils [12, 13].

Address for correspondence: Hilal Kaya Erdogan MD, Department of Dermatology, Eskişehir Osmangazi University, Buyukdere District, Osmangazi University Meselik Campus 26040 Eskisehir, Turkey, phone: +90 222239 29 79-3551, 05057161770, fax: +90 02222393772 , e-mail: hilalkayaerdogan@yahoo.com

Received: 6.02.2017, accepted: 2.05.2017. 
Aim

In our study, we aimed to evaluate the oxidative stress status in rosacea patients by measuring serum TAS, TOS, oxidative stress index (OSI) and AOPP levels.

\section{Material and methods}

Our study included 70 rosacea patients and 30 healthy volunteers aged between 18 and 65 years. Patients and volunteers with systemic inflammatory disease, having any systemic treatment, antioxidants, and food and vitamin supplements within the last month, patients with any other skin disorders were excluded. In addition, smoking and alcohol consumption, pregnancy and nursing were also among exclusion criteria.

The ethics committee approved the study protocol. Patients and volunteers were informed and informed consent form was signed before being admitted to the study.

The blood samples were centrifuged at $3000 \mathrm{~g}$ for 20 min within 30 min following sampling and the separated serum was stored at $-80^{\circ} \mathrm{C}$ up to the day when measurements were done. Total antioxidant status and TOS measurements were done colorimetrically by Roche/Hitachi Modular (Mannheim, Germany) auto-analyzer. Total antioxidant status was measured by using commercial kits of Rel Assay (Rel Assay Kit Diagnostics, Turkey) developed by Erel [11]. Trolox, water-soluble analogue of vitamin E, was used as a calibrator. Results were expressed as mmol Trolox equiv./lt. The TOS level was measured by using commercial kits of Rel Assay (Rel Assay Kit Diagnostics, Turkey) developed by Erel [10]. Hydrogen peroxide was used as a calibrator. Results were expressed as $\mu \mathrm{mol} \mathrm{H}_{2} \mathrm{O}_{2}$ equiv./lt. The percentage expression of the ratio of TOS level to TAS level was calculated as OSI. Results were expressed as an "arbitrary unit" $(\mathrm{AU})$ and calculated according to the following formula: $\mathrm{OSI}=\mathrm{TOS}\left(\mu \mathrm{mol} \mathrm{H}_{2} \mathrm{O}_{2}\right.$ equiv./It)/TAS, mmol Trolox equiv./ It $\times 10$. The serum AOPP level was measured with competitive enzyme linked immunosorbent assay (ELISA, cat. no: MBS028634, MyBioSource, San Diego, USA).

\section{Statistical analysis}

SPSS 22.0 (IBM Corp. Released 2013. IBM SPSS Statistics for Windows, Version 22.0. Armonk, NY: IBM Corp.) software was used for data analysis. Continuous quantitative data were expressed as $n$, mean and standard deviation and qualitative data were expressed as $n$ (\%), median, quartile 1 and quartile 3 (percentile 25-75 respectively). The Shapiro-Wilk test was used to observe whether the data showed normal distribution. In the comparison of groups that showed normal distribution, independent samples $t$-test was performed where there were two groups, and one-way ANOVA was used where the number of groups was three and more. In the comparison of groups that did not show normal distribution,
Mann Whitney $U$ Test was used where the number of groups was two, Kruskal-Wallis $\mathrm{H}$ test was used where the number of groups was three and more. In order to demonstrate the correlation between variables, Spearman's Correlation test was done for variables without normal distribution results. Fisher's exact $\chi^{2}$ test was applied for a categorical data set. Probability value of $p<0.05$ was considered as significant.

\section{Results}

The study included 70 patients with rosacea (63 females and 7 males) and a control group of 30 healthy volunteers (27 females and 3 males). Mean age of patients with rosacea was $42.61 \pm 13.02$ years and mean age for the control group was $40.33 \pm 11.50$ years. Rosacea and control groups were both age- and gender-matched ( $p=1.000, p=0.406)$.

The disease duration in the rosacea group was $0.5-40$ (mean: 8.3) years. The Fitzpatrick's skin type was type II in 25 (35.7\%), type III in 34 (48.6\%) and type IV in 11 (15.7\%) patients. Papulopustular rosacea was seen in 48 (68.6\%) and erythematotelangiectatic rosacea in 22 (31.4\%) patients. Ocular involvement was found in 14 (20\%) patients. Overall, $85.7 \%$ of the patients had at least one symptom. The symptoms included burning in 46 (65.7\%), itching in 40 (57.1\%), stinging in 19 (27.1\%) and pain in 16 (22.9\%) cases. The most common triggering factor was stress in 64 (91.4\%) patients; the other factors were hot weather in $58(82.9 \%)$, sun exposure in $53(75.7 \%)$, cold weather in $42(60 \%)$, physical exercise in 39 (60\%), wind in 34 (48.6\%), spicy foods in 27 (38.6\%), hot beverages in 23 (32.9\%) and alcohol in 4 (5.7\%) patients.

When TAS, TOS, OSI and AOPP levels were compared between rosacea and control groups, there was no difference for OSI levels; while TAS, TOS and AOPP levels were significantly higher in the rosacea group $(p=0.151$, $p=0.013, p=0.034, p=0.017$, respectively) (Table 1 ).

Table 1. Comparison of TAS, TOS, OSI and AOPP levels in the patient and control groups

\begin{tabular}{|c|c|c|c|}
\hline \multirow[t]{2}{*}{ Parameter } & \multicolumn{2}{|c|}{$\begin{array}{c}\text { Mean } \pm \text { standard deviation } \\
\text { Median }(\mathrm{Q} 1-\mathrm{Q} 3)\end{array}$} & \multirow[t]{2}{*}{$P$-value } \\
\hline & Controls & Patients & \\
\hline TAS & $\begin{array}{c}2.26 \pm 0.27 \\
2.24(2.01-2.45)\end{array}$ & $\begin{array}{c}2.40 \pm 0.25 \\
2.44(2.21-2.60)\end{array}$ & $0.013^{*}$ \\
\hline TOS & $\begin{array}{c}2.96 \pm 1.01 \\
3.02(2.15-3.52)\end{array}$ & $\begin{array}{c}3.67 \pm 1.89 \\
3.26(2.65-4.46)\end{array}$ & $0.034^{\dagger}$ \\
\hline AOPP & $\begin{array}{c}63.87 \pm 49.06 \\
49.92(23.25-108.61)\end{array}$ & $\begin{array}{c}101.33 \pm 73.12 \\
92.39(43.70-149.42)\end{array}$ & $0.017^{\dagger}$ \\
\hline OSI & $\begin{array}{c}12.99 \pm 3.64 \\
12.87(10.64-15.68)\end{array}$ & $\begin{array}{c}15.24 \pm 6.92 \\
13.86(11.06-17.80)\end{array}$ & $0.151^{\dagger}$ \\
\hline
\end{tabular}

${ }^{*}$ Independent samples $t$-test, ${ }^{\dagger}$ Mann-Whitney $U$ test, TAS - total antioxidant status, TOS - total oxidant status, OSI - oxidative stress index, AOPP - advanced oxidation protein products. 
Table 2. Correlation between TAS, TOS, OSI and AOPP levels and the disease duration

\begin{tabular}{llllll}
\hline & & TAS & TOS & AOPP & OSI \\
\hline Disease duration & $r$ & 0.043 & 0.068 & 0.053 & 0.063 \\
\cline { 2 - 6 } & $p$ & 0.723 & 0.576 & 0.664 & 0.603 \\
\hline
\end{tabular}

*Spearman's correlation test.

Table 3. Comparison of TAS, TOS, OSI and AOPP levels in the patient group according to family history, rosacea type, symptoms and ocular involvement (mean \pm standard deviation, median (Q1-Q3))

\begin{tabular}{|c|c|c|c|c|}
\hline \multirow[t]{2}{*}{ Parameter } & \multicolumn{3}{|c|}{ Family history } & \multirow[t]{2}{*}{$P$-value } \\
\hline & Negative & & Positive & \\
\hline \multirow[t]{2}{*}{ TAS } & $2.40 \pm 0.26$ & & $2.41 \pm 0.25$ & \multirow[t]{2}{*}{$0.936^{*}$} \\
\hline & $2.41(2.21-2.60)$ & & $2.46(2.20-2.59)$ & \\
\hline \multirow[t]{2}{*}{ TOS } & $3.72 \pm 2.18$ & & $3.58 \pm 1.18$ & \multirow[t]{2}{*}{$0.862^{\dagger}$} \\
\hline & $3.20(2.63-4.52)$ & & $3.42(2.64-4.17)$ & \\
\hline \multirow[t]{2}{*}{ AOPP } & $105.23 \pm 68.42$ & & $93.87 \pm 82.41$ & \multirow[t]{2}{*}{$0.287^{\dagger}$} \\
\hline & 93.85 (46.76-161.78) & & 72.93 (41.49-111.15) & \\
\hline \multirow[t]{2}{*}{ OSI } & $15.47 \pm 8.02$ & & $14.78 \pm 4.17$ & \multirow[t]{2}{*}{$0.911^{\dagger}$} \\
\hline & 13.60 (10.98-18.64) & & $14.22(11.45-17.31)$ & \\
\hline \multirow[t]{2}{*}{ Parameter } & \multicolumn{3}{|c|}{ Rosacea type } & \multirow[t]{2}{*}{$P$-value } \\
\hline & Erythematotelangiectatic & & Papulopustular & \\
\hline \multirow[t]{2}{*}{ TAS } & $2.35 \pm 0.28$ & & $2.43 \pm 0.24$ & \multirow[t]{2}{*}{$0.266^{\star}$} \\
\hline & $2.35(2.13-2.59)$ & & $2.47(2.22-2.61)$ & \\
\hline \multirow[t]{2}{*}{ TOS } & $3.75 \pm 1.44$ & & $3.64 \pm 2.07$ & \multirow[t]{2}{*}{$0.519^{\dagger}$} \\
\hline & $3.63(2.51-4.87)$ & & $3.17(2.67-4.22)$ & \\
\hline \multirow[t]{2}{*}{ AOPP } & $110.21 \pm 94.57$ & & $97.26 \pm 61.63$ & \multirow[t]{2}{*}{$0.980^{\dagger}$} \\
\hline & $83.32(36.60-175.93)$ & & 92.39 (46.46-128.28) & \\
\hline \multirow[t]{2}{*}{ OSI } & $15.81 \pm 5.38$ & & $14.97 \pm 7.56$ & \multirow[t]{2}{*}{$0.250^{\dagger}$} \\
\hline & 15.85 (11.29-19.88) & & 13.24 (11.01-17.11) & \\
\hline \multirow[t]{2}{*}{ Parameter } & \multicolumn{3}{|c|}{ Symptoms } & \multirow[t]{2}{*}{$P$-value } \\
\hline & No symptom & Only one symptom & More than one symptom & \\
\hline \multirow[t]{2}{*}{ TAS } & $2.36 \pm 0.30$ & $2.38 \pm 0.23$ & $2.43 \pm 0.26$ & \multirow[t]{2}{*}{$0.647^{\ddagger}$} \\
\hline & $2.39(2.11-2.60)$ & $2.42(2.21-2.58)$ & $2.49(2.21-2.62)$ & \\
\hline TOS & $4.31 \pm 4.09$ & $3.33 \pm 1.32$ & $3.74 \pm 1.17$ & $0.317^{\S}$ \\
\hline & $3.12(2.68-3.86)$ & $3.10(2.39-3.90)$ & $3.45(2.96-4.80)$ & \\
\hline AOPP & $124.02 \pm 58.03$ & $98.07 \pm 73.25$ & $97.18 \pm 77.42$ & $0.296^{\S}$ \\
\hline & 111.24 (86.94-178.36) & 73.06 (42.62-138.21) & 93.54 (33.95-134.35) & \\
\hline OSI & $17.39 \pm 13.72$ & $14.01 \pm 5.30$ & $15.49 \pm 5.00$ & $0.528^{\S}$ \\
\hline & 13.28 (11.99-17.51) & 13.08 (10.21-16.95) & 13.98 (11.75-19.73) & \\
\hline Parameter & & Ocular involvement & & $P$-value \\
\hline & Negative & & Positive & \\
\hline TAS & $2.38 \pm 0.25$ & & $2.48 \pm 0.25$ & $0.179^{\star}$ \\
\hline & $2.42(2.20-2.58)$ & & 2.47 (2.33-2.69) & \\
\hline TOS & $3.70 \pm 2.06$ & & $3.58 \pm 0.98$ & $0.758^{\dagger}$ \\
\hline & $3.26(2.58-4.47)$ & & $3.34(2.85-4.33)$ & \\
\hline AOPP & $101.02 \pm 73.17$ & & $102.57 \pm 75.64$ & $0.883^{\dagger}$ \\
\hline & 93.56 (41.15-155.57) & & $78.91(53.41-113.40)$ & \\
\hline OSI & $15.48 \pm 7.59$ & & $14.27 \pm 3.06$ & $0.883^{\dagger}$ \\
\hline & 13.70 (11.01-18.33) & & 14.19 (11.56-15.65) & \\
\hline
\end{tabular}

*Independent samples $t$-test, ${ }^{\dagger}$ Mann-Whitney $U$ test, ${ }^{\star}$ One way analysis of variance analysis, ${ }^{\S} \mathrm{Kruskal}$ Wallis $\mathrm{H}$ test. 
In the rosacea group, there was no correlation between TAS, TOS, OSI and AOPP levels and disease duration (Table 2). There was no significant difference between family history, rosacea type, symptom frequency and ocular involvement and TAS, TOS, OSI and AOPP levels in the rosacea group (Table 3).

\section{Discussion}

Reactive oxygen species (ROS) may play a role in many dermatologic disorders by causing inflammation, photosensitivity, ageing and tumor development. To protect organisms from oxidative stress, there are some innate defense (antioxidant) mechanisms [7].

Rosacea is a chronic skin disease with unknown origin; but it is thought that inflammation plays a central role in the pathogenesis of rosacea. Inflammation is associated with other factors such as UV radiation, vascular changes and oxidative tissue damage. Oxidative stress can play a role in the pathogenesis of rosacea by several mechanisms: via neutrophils by producing ROS, oxidative modification of proteins and lipids by ROS, alterations in lipid balance, antimicrobial peptides (cathelicidin LL-37), cytokines and other inflammatory mediators (IL-1 and TNF- $\alpha$ ) [1].

Glutathione-S-transferases (GST) are proteins that detoxify toxic products produced by UV-related oxidative stress. It is thought that GSTM1 and GSTT1 null phenotype carriers are sensitive to oxidative stress and they develop more severe inflammation after UV exposure. In a study investigating genetic susceptibility to oxidative stress and rosacea, it is hypothesized that GSTs gene polymorphism can play a role in rosacea pathogenesis by increasing ROS levels and decreasing antioxidant potential. Yazıcı et al. found a significant association between the GSTT1 and/or GSTM1 null genotypes and rosacea [6].

Paraoxonase-1 (PON1) is an antioxidant enzyme and it has paraoxonase, arylesterase and dyazoxonase activities. Lipid hydroperoxide $(\mathrm{LOOH})$ is a lipid peroxidation marker that shows the oxidative stress. Takçı et al. found that serum paraoxonase and arylesterase activities were lower and the serum $\mathrm{LOOH}$ level was higher in rosacea patients than controls. They suggested that these results support the role of oxidative stress in rosacea pathogenesis [4].

Iron in the cells which was produced by proteolysis of ferritin and oxidative damage can play a role in rosacea. Tisma et al. found that serum peroxide levels, ferritinpositive cells were higher and total antioxidative potential levels were lower in rosacea. Finally they showed a decrease in the systemic antioxidant defense system and systemic oxidative stress in rosacea [5]. Moreover Oztas et al. evaluated the role of free oxygen radicals in the etiopathogenesis of rosacea. They found that while in the mild rosacea, antioxidant activity increased to protect the skin; in severe rosacea, the antioxidant defense system decreased. They concluded that the antioxidant systems resist at the beginning but become insufficient in severe disease [7]. In our study, we found that antioxidant status increased. We think that antioxidant capacity can increase in response to increased oxidant status.

There are studies investigating the relationship between Helicobacter pylori (HP), oxidative stress and rosacea $[8,9]$. Helicobacter pylori can contribute rosacea pathogenesis by increasing the nitric oxide (NO) levels and causing vasodilatation, inflammation, and immunomodulation. Gürer et al. put forward that HP colonizes the gastric mucosa and increases the free oxygen radicals and proinflammatory cytokines in rosacea patients. They found the high HP seropositivity in rosacea patients; but the NO levels were normal. NO associated with HP was not found to play a role in inflammation of rosacea in their study [8]. Baz et al. evaluated the plasma ROS activity and antioxidant potential (AOP) and their relation with HP in rosacea. They showed ROS increased and AOP decreased in rosacea patients independently of HP [9].

In accordance with other studies, we found that oxidative stress was significantly higher in rosacea patients. We also showed that antioxidant status was increased in rosacea unlike the results of other studies. Antioxidant status may be increased to protect the skin from oxidative stress as a compensatory mechanism. In other words, antioxidant capacity may increase in response to increased oxidant status.

Reduction of oxidative stress can be tried for the treatment of rosacea. Drugs such as tetracyclines, metronidazole and retinoids have antioxidant activities. They decrease neutrophil-generated ROS. Bakar et al. evaluated the antioxidant effects of azithromycin on ROS in rosacea. At the end of one-month azithromycin therapy, ROS levels decreased significantly. Some antioxidants such as vitamin $C, E$, and ubiquinone also can be used to decrease oxidative stress $[3,5,7]$.

\section{Conclusions}

We observed that serum TAS, TOS and AOPP levels were significantly higher in rosacea patients, but there was no significant difference among the disease activity parameters. While the TAS and TOS levels show global oxidative status; the AOPP reflects the protein oxidation derived from neutrophils/monocytes. These results can support the role of oxidative stress in the pathogenesis of rosacea. However, further studies are warranted in order to clarify the direction of the relation, whether the imbalance of oxidant/antioxidant status is a result or a cause of inflammation in rosacea.

\section{Acknowledgments}

This study was supported by the Turkish Dermatology Association. This study was conducted in the Eskisehir 
Osmangazi University Faculty of Medicine, Department of Dermatology.

\section{Conflict of interest}

The authors declare no conflict of interest.

\section{References}

1. Jones DA. Rosacea, reactive oxygen species, and azelaic acid. J Clin Aesthet Dermatol 2009; 2: 26-30.

2. Woo YR, Lim JH, Cho DH, Park HJ. Rosacea: molecular mechanisms and management of chronic cutaneous inflammatory condition. Int J Mol Sci 2016; 17: E1562.

3. Bakar O, Demirçay Z, Yuksel M, et al. The effect of azithromycin on reactive oxygen species in rosacea. Clin Exp Dermatol 2007; 32: 197-200.

4. Takci Z, Bilgili SG, Karadag AS, et al. Decreased serum paraoxonase and arylesterase activities in patients with rosacea. J Eur Acad Dermatol Venereol 2015; 29: 367-70.

5. Tisma VS, Basta-Juzbasic A, Jaganjac M, et al. Oxidative stress and ferritin expression in the skin of patients with rosacea. J Am Acad Dermatol 2009; 60: 270-6.

6. Yazici AC, Tamer L, Ikizoglu G, et al. GSTM1 and GSTT1 null genotypes as possible heritable factors of rosacea. Photodermatol Photoimmunol Photomed 2006; 22: 208-10.

7. Oztas MO, Balk M, Ogüs E, et al. The role of free oxygen radicals in the aetiopathogenesis of rosacea. Clin Exp Dermatol 2003; 28: 188-92.

8. Gürer MA, Erel A, Erbaş D, et al. The seroprevalence of Helicobacter pylori and nitric oxide in acne rosacea. Int I Dermatol 2002; 41: 768-70.

9. Baz K, Cimen MY, Kokturk A, et al. Plasma reactive oxygen species activity and antioxidant potential levels in rosacea patients: correlation with seropositivity to Helicobacter pylori. Int I Dermatol 2004; 43: 494-7.

10. Erel O. A new automated colorimetric method for measuring total oxidant status. Clin Biochem 2005; 38: 1103-11.

11. Erel O. A novel automated direct measurement method for total antioxidant capacity using a new generation, more stable ABTS radical cation. Clin Biochem 2004; 37: 277-85.

12. Witko-Sarsat V, Friedlander M, Capeillère-Blandin C, et al. Advanced oxidation protein products as a novel marker of oxidative stress in uremia. Kidney Int 1996; 49: 1304-13.

13. Yazici C, Köse K, Caliş M, et al. Increased advanced oxidation protein products in Behçet's disease: a new activity marker? Br J Dermatol 2004; 151: 105-11. 